\title{
Procaspase-3 activation by a metalloprotease secreted from Vibrio vulnificus
}

\author{
HYO YOUNG KIM ${ }^{1}$, ALAN K. CHANG ${ }^{1}$, JUNG EUN PARK ${ }^{1,2}$, IL-SEON PARK ${ }^{1}$, \\ SEONG MYEONG YOON ${ }^{3}$ and JUNG SUP LEE LE, $^{1,2,4}$ \\ ${ }^{1}$ Research Center for Proteineous Materials, ${ }^{2}$ BK21 Research Team for Protein Activity Control, Departments of \\ ${ }^{3}$ Marine Life Science, ${ }^{4}$ Biotechnology, Chosun University, Gwangju 501-759, Korea
}

Received May 10, 2007; Accepted June 29, 2007

\begin{abstract}
Vibrio vulnificus is a marine bacterium and a human pathogen capable of causing wound infection and septicemia. We previously showed that the metalloprotease vEP secreted by $V$. vulnificus activates prothrombin in vitro. To further investigate the ability of vEP to activate other zymogens, we used a mutant form of procaspase- 3 which lacks the native cleavage sites as a zymogen. The mutant zymogen was activated by vEP to yield a mature enzyme with a maximum increase in caspase- 3 activity of approximately 14-fold in a time-dependent manner. However, the increase started to decline with prolonged incubation and with higher protease concentration as a result of further cleavage of the mature enzyme. Western blot analysis revealed a band of $\sim 17 \mathrm{kDa}$ for the cleavage product, which corresponded with the change in caspase- 3 activity. The activated procaspase- 3 by vEP was also able to cleave poly(ADP-ribose) polymerase in a cell-free system, and was inhibited by Ac-DEVD-CHO, a potent caspase- 3 inhibitor. The results presented are the first to demonstrate the in vitro activation of one of the crucial enzymes involved in cell death by a bacterial extracellular metalloprotease.
\end{abstract}

\section{Introduction}

Vibrio vulnificus (V. vulnificus) is an opportunistic human pathogen causing wound infection and septicemia $(1,2)$. It secretes a zinc metalloprotease that has been reported to have

Correspondence to: Professor Jung Sup Lee, Department of Biotechnology, College of Natural Sciences, Chosun University, Gwangju 501-759, Korea

E-mail: jsplee@mail.chosun.ac.kr

Abbreviations: CAPS, 3-(cyclohexylamino)-1-propanesulfonic acid; DTT, dithiothreitol; EDTA, ethylenediaminetetraacetic acid; EGTA, ethylene glycol-bis(2-aminoethylether)-N,N.N',N'tetraacetic acid; PARP, poly(ADP-ribose) polymerase; PBS, phosphate-buffered saline; PMSF, phenylmethanesulfonyl fluoride; $p \mathrm{NA}, p$-nitroaniline; PVDF, polyvinylidene fluoride

Key words: Vibrio vulnificus, metalloprotease, procaspase-3 many biological functions. These include the degradation of various plasma proteins and vascular permeability enhancement through the generation of inflammatory mediators. The wide spectrum of its activities in an in vitro system has made it a very interesting protease. It has not yet been conclusively demonstrated whether these biological activities of the enzyme contribute to the pathogenicity exerted by the bacterium $V$. vulnificus, although it has been shown that injection of the purified enzyme into animals produced some of the pathogenic symptoms observed with the bacterial infection $(3,4)$. We previously reported that the protease secreted by $V$. vulnificus, ATCC 29307, which we named $\mathrm{vEP}$, is a broad specificity enzyme that activates prothrombin in vitro (5). The cleavage of prothrombin produced a number of fragments and one of these corresponded to that of $\alpha$ thrombin as shown by Western blot analysis. The cleavage site occurred at $\mathrm{Thr}^{272}-\mathrm{Ala}^{273}$, which is very near the site $\left(\mathrm{Arg}^{271}-\mathrm{Thr}^{272}\right)$ recognized by Factor $\mathrm{Xa}$, the physiological prothrombin activator. Based on this property, we speculated that vEP might also activate the zymogens of other proteases if it cleaves these zymogens at sites that are very near to the native cleavage sites for their activation.

Treatment of HeLa and NIH3T3 cells with vEP resulted in cell death (unpublished data). However, the mode of cell death exerted by vEP has not yet been established, and so far there is no evidence that this enzyme causes direct apoptosis, which is a tightly regulated cellular event that plays a critical role in the development and tissue homeostasis of metazoans $(6,7)$. At the molecular level, apoptosis is tightly controlled and is mainly orchestrated by the activation of the aspartatespecific cysteine proteases known as caspases $(8,9)$. The degradation of certain proteins by members of the caspase family is a general event taking place in cells undergoing apoptosis. The most prevalent caspase in the cell is caspase- 3 . It is a mediator caspase that is ultimately responsible for the majority of apoptotic effects. Inhibition of caspase- 3 or caspase-3-like proteases in various cells has been shown to block apoptosis $(10,11)$. In addition to its role in cell death, caspase-3 is important for survival, as caspase-3-knockout mice are born at a low frequency and die after only a few weeks (12).

Like other caspases, caspase-3 is initially synthesized as an inactive 32-kDa precursor (procaspase-3). During activation, procaspase- 3 is converted to $20-\mathrm{kDa}$ and $12-\mathrm{kDa}$ 
peptides via proteolysis at the cleavage site, Ile ${ }^{172}-\mathrm{Glu}-\mathrm{Thr}-$ Asp $\downarrow \operatorname{Ser}^{176}$ (arrow indicates the cleavage site). The $20-\mathrm{kDa}$ peptide undergoes further cleavage at the site, Glu $^{25}$-Ser-MetAsp $\downarrow \operatorname{Ser}^{29}$ (arrow indicates the cleavage site), generating the $17-\mathrm{kDa}$ peptide (13). The active caspase-3 is a tetrameric protein consisting of two p17 and two p12 subunits. Recent reports have shown that procaspase- 3 is catalytically active with lower $k_{\text {cat }}$ and similar $K_{\mathrm{m}}$ compared to that of caspase-3 $(14,15)$. In these reports, an uncleavable procaspase-3 mutant (D9A, D28A, or D175A) was used instead of natural procaspase-3. Using the same procaspase-3 [procaspase-3(D3A)], which is incapable of auto-activation when expressed in E. coli, we were able to show that vEP could activate this caspase- 3 zymogen to generate mature caspase- 3 which exhibited the same substrate and inhibitor specificity as wild-type caspase-3 in a cell-free system.

\section{Materials and methods}

Materials. Ac-DEVD-pNA and Ac-DEVD-CHO were purchased from Bachem. Mouse monoclonal anti-caspase-3 antibody was obtained from Sigma. Rabbit polyclonal antiPARP antibody was obtained from Santa Cruz Biotechnology. Anti-rabbit and anti-mouse IgGs were obtained from Jackson Immunoresearch. $\mathrm{Ni}^{2+}$-NTA was purchased from Amersham Biosciences. All other chemicals used were obtained from Sigma.

Enzyme expression and purification. The construction of procaspase-3(D3A) has been previously described (15). The aspartate at residues 9,28 and 175 of the procaspase coding region was substituted with alanine to prevent autoprocessing of the zymogen. The enzyme contained a His tag at the $\mathrm{N}$ - and C-termini. Procaspase-3(D3A) will henceforth be referred to as D3A for simplicity. D3A was expressed in the BL21(DE3) E. coli strain (Novagen), and protein induction was carried out with $0.2 \mathrm{mM}$ IPTG at $25^{\circ} \mathrm{C}$ overnight. The enzyme was purified from a Ni-NTA column and finally exchanged into a buffer containing $25 \mathrm{mM}$ Tris- $\mathrm{HCl}, \mathrm{pH} 7.5$ and $40 \%$ glycerol and stored in small aliquots at $-70^{\circ} \mathrm{C}$. vEP was purified from the culture supernatant of $V$. vulnificus ATCC 29307 as described previously (5).

Activation of D3A by $v E P$. Activation of D3A was performed by incubating the zymogen $(0.16-0.58 \mathrm{mg} / \mathrm{ml})$ with $0-8 \mu \mathrm{g} / \mathrm{ml}$ vEP in $25 \mathrm{mM}$ Tris- $\mathrm{HCl}, \mathrm{pH} 7.5$ at room temperature. The reaction was terminated by addition of $1 \mathrm{mM} 1,10$ phenanthroline, a potent inhibitor of vEP (5) and then subjected to SDS-PAGE or Western blot analysis with anticaspase-3 antibody or activity assay with the caspase-3specific chromogenic substrate, Ac-DEVD- $p$ NA in a $100 \mu 1$ reaction volume consisting of $20 \mathrm{mM}$ HEPES, pH 7.5, $1 \mathrm{mM}$ EDTA, $1 \mathrm{mM}$ EGTA, $20 \mathrm{mM} \mathrm{NaCl}, 10 \mathrm{mM}$ DTT and $1 \mathrm{mM}$ 1,10 -phenanthroline. The release of $p \mathrm{NA}$ was continuously monitored by absorbance at $405 \mathrm{~nm}$ at $37^{\circ} \mathrm{C}$ using a 96-well plate reader (Molecular Devices). For assay with the inhibitor Ac-DEVD-CHO, $0.5 \mathrm{mg} / \mathrm{ml}$ zymogen was activated with $2 \mu \mathrm{g} /$ $\mathrm{ml}$ vEP for $10 \mathrm{~min}$ at room temperature and then terminated by the addition of $1 \mathrm{mM}$ 1,10-phenanthroline. Aliquots were extracted and assayed for caspase- 3 activity as described above in the absence or presence of various concentrations of the inhibitor.

Cleavage of PARP. To prepare the cell lysate, NIH3T3 cells from two $10 \mathrm{~cm}$ dishes were harvested, washed twice with PBS, re-suspended in $100 \mu 1$ of lysis buffer (10 mM Tris$\mathrm{HCl}, \mathrm{pH} 7.5,0.5 \%$ Triton $\mathrm{X}-100,5 \mathrm{mM}$ EDTA, $1 \mathrm{mM}$ PMSF) and then incubated on ice on a shaker for $15 \mathrm{~min}$. Ten $\mu 1$ of the lysate (150 $\mu \mathrm{g}$ protein) was mixed with $10 \mu \mathrm{l}$ of vEP-activated D3A (as described above) for different time periods at room temperature, and the reaction was terminated by the addition of $20 \mu 1$ of $2 \mathrm{X}$ SDS-PAGE sample buffer. The samples were heated at $100^{\circ} \mathrm{C}$ for $1 \mathrm{~min}$ and then subjected to SDS-PAGE with subsequent Western blot analysis using an antibody against PARP.

SDS-PAGE and Western blot analysis. SDS-PAGE was performed according to the method of Laemmli (16). Samples to be analyzed were mixed with an equal volume of $2 \mathrm{X}$ SDSPAGE sample buffer and heated at $100^{\circ} \mathrm{C}$ for $1-2$ min, and then loaded onto $15 \%$ gel. After electrophoresis, protein bands were visualized by staining the gel with Coomassie blue. For Western blot analysis, protein samples were subjected to SDS-PAGE and then transferred to a PVDF membrane. The membrane was blocked at room temperature for $2 \mathrm{~h}$ with blocking solution (PBS containing 5\% skim milk and $0.2 \%$ Tween-20) and then incubated for $1 \mathrm{~h}$ with either mouse monoclonal anti-caspase-3 antibody diluted 1:5000 or with rabbit polyclonal anti-PARP antibody diluted 1:500. The blot was washed and incubated with a 1:5000 dilution of peroxidase-conjugated anti-rabbit or anti-mouse IgG antibody for $1 \mathrm{~h}$. The blot was washed, treated with ECL Western Blotting Detection reagents (Amersham Biosciences), and then exposed to Hyperfilm ECL (Amersham Biosciences).

$N$-terminal sequencing. Protein samples were subjected to electrophoresis on $15 \%$ SDS-PAGE gel. After electrophoresis, proteins were transferred to a PVDF membrane in $10 \mathrm{mM}$ 3-(cyclohexylamino)-1-propanesulfonic acid (CAPS) buffer, pH 11.0 containing $10 \%$ methanol. The blot was stained with Coomassie blue, followed by destaining. Target bands were excised from the blot and subjected to N-terminal sequencing using an Applied Biosystem Precise sequencer (Applied Biosystem).

\section{Results}

Activation of D3A. The substitution of Ala for Asp in the cleavage sites of D3A prevented the zymogen activation since simple expression of wild-type caspase zymogens in $E$. coli usually results in their activation $(17,18)$. The zymogen itself showed some activity, but the activity clearly varied with the concentrations of vEP used during the activation process, with a maximum increase of $\sim 14$-fold over the zymogen at $2 \mu \mathrm{g} /$ $\mathrm{ml}$ vEP (Fig. 1A). vEP exhibited no detectable activity toward Ac-DEVD- $p$ NA (data not shown). At higher concentrations of $\mathrm{vEP}$, the activity decreased probably due to additional cleavage of the mature enzyme by vEP. At a fixed concentration of vEP $(2 \mu \mathrm{g} / \mathrm{ml})$, the caspase- 3 activity of activated D3A also exhibited an increase that paralleled the 
A

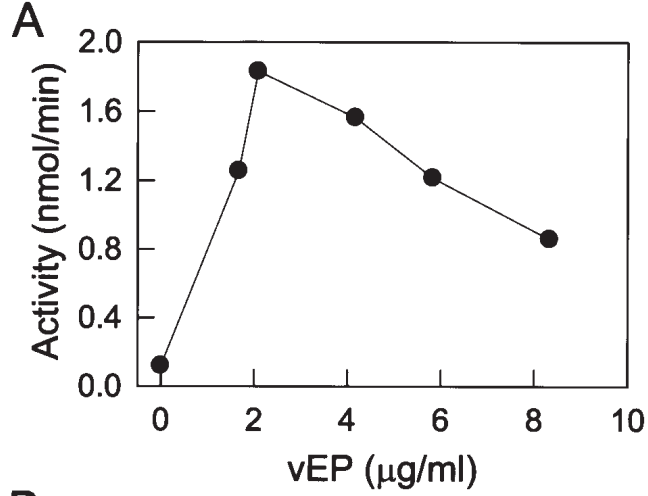

B

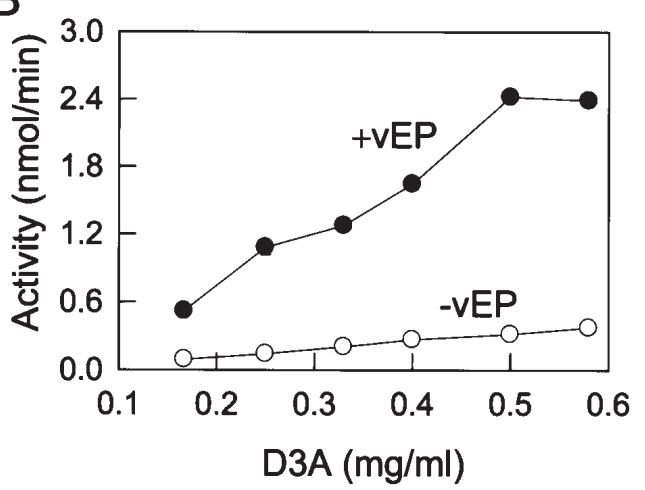

Figure 1. Effect of vEP and zymogen concentration on the activation of D3A. (A) D3A $(0.5 \mathrm{mg} / \mathrm{ml})$ was activated with different concentrations of vEP at room temperature for $10 \mathrm{~min}$. (B) Different concentrations of D3A were activated with $2 \mu \mathrm{g} / \mathrm{ml}$ vEP at room temperature for $10 \mathrm{~min}$. Caspase-3 activity was assayed with Ac-DEVD- $p$ NA as described in Materials and methods. Data are the means of two experiments performed in duplicate.

concentrations of zymogen used in the activation reaction, but the activity appeared to reach a maximum at $0.5 \mathrm{mg} / \mathrm{ml}$ zymogen (Fig. 1B). At $0.5 \mathrm{mg} / \mathrm{ml} \mathrm{zymogen} \mathrm{and} 2 \mu \mathrm{g} / \mathrm{ml}$ $\mathrm{vEP}$, the activity of caspase- 3 increased with increasing activation time reaching a peak at $10 \mathrm{~min}$ and then started to decline with increasing activation time (Fig. 2A). The loss of activity correlated with the loss of a band that was approximately the same size as the p17 subunit of mature caspase-3 as revealed by Western blot analysis (Fig. 2B), and at the same time resulted in the accumulation of a band of $\sim 14 \mathrm{kDa}$, which could be derived from the cleavage of the larger band via further proteolysis by vEP. This band did not belong to the p12 subunit since the anti-caspase- 3 antibody reacted only with the 17 subunit. The band corresponding to 24-kDa appeared to be quite stable over a 40 min incubation period, whereas much of the zymogen had disappeared by this time point. The disappearance of the zymogen with time did not result in a corresponding increase for the mature enzyme, suggesting that cleavage of the zymogen to yield active caspase- 3 was transient and the active caspase-3, once formed, was highly susceptible to further cleavage by vEP.

Since vEP-activated D3A probably consisted of more than one species with activity toward DEVD, no kinetic properties were determined for the mature enzyme. However, the $K_{\mathrm{m}}$ for Ac-DEVD-pNA determined from the mixed species was $30.5 \mu \mathrm{M}$ compared with the values of 2.0-16.8 $\mu \mathrm{M}$ reported for the recombinant wild-type mature caspase- 3
A
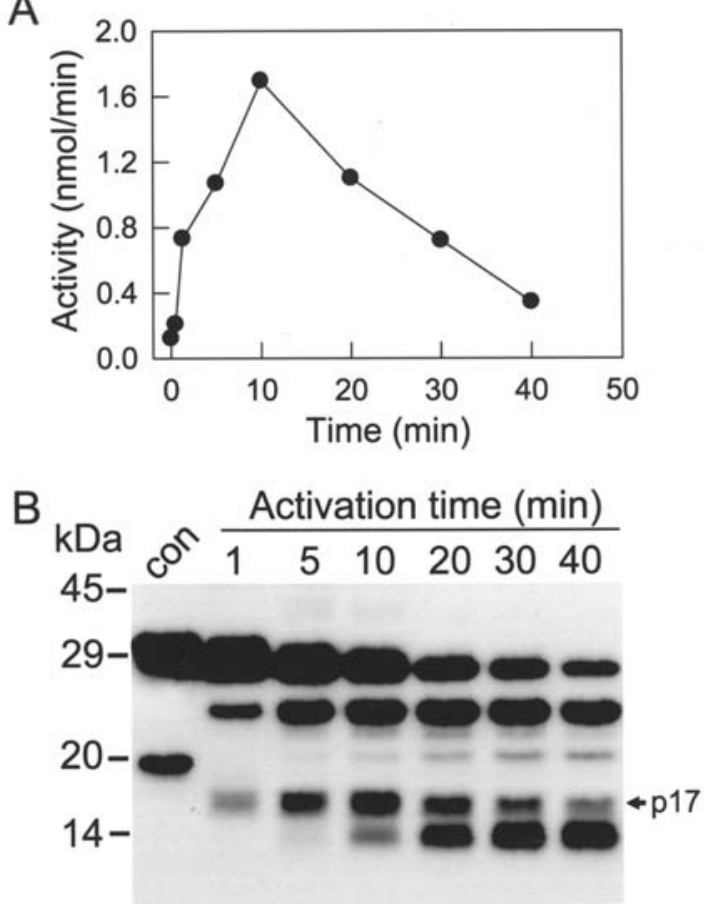

Figure 2. Time-dependent activation of D3A by vEP. D3A $(0.5 \mathrm{mg} / \mathrm{ml})$ was activated by vEP $(2 \mu \mathrm{g} / \mathrm{ml})$ at room temperature for different time intervals and then subjected to activity assay toward Ac-DEVD-pNA (A) and Western blot analysis (B). The arrow in B indicates the position of the p17 subunit of mature caspase- 3 .

$(14,15,19)$. The $K_{\mathrm{m}}$ for D3A $(12.8 \mu \mathrm{M})$ was similar to the reported value of $13.2 \mu \mathrm{M}(15)$. The inhibitory effect of AcDEVD-CHO on vEP-activated D3A was also confirmed, with an apparent $K_{\mathrm{i}}$ of $18.3 \mathrm{nM}$ at $0.2 \mathrm{mM}$ substrate.

Cleavage fragments of D3A. From the cleavage of D3A, it seemed clear that vEP cleaved at multiple sites within the zymogen and the rate of cleavage for each site might differ. The purified zymogen consisted mainly of a $32-\mathrm{kDa}$ protein on SDS-PAGE with minor contaminating bands of $\sim 20 \mathrm{kDa}$ and an even fainter band near the 17-kDa regions (Fig. 3A). Both bands could be a product of proteolysis by $E$. coli proteases during expression and/or purification. Cleavage of the zymogen by vEP yielded various fragments, with a $24-\mathrm{kDa}$ fragment being the most abundant. Various fragments of 18$12 \mathrm{kDa}$ that were absent in the case of non-cleaved zymogen were present at a low amount. These fragments covered the size of both the large and small subunits of the mature caspase-3. With the non-activated D3A, Western blotting detected a band of $\sim 20 \mathrm{kDa}$ in addition to the zymogen (Fig. 3B); therefore, this fragment appeared to be caspase-3 protein derived probably from the cleavage of the zymogen during expression or purification. Notably, at longer activation times, the cleavage of the zymogen may proceed with higher non-specificity without yielding active mature caspase-3. Two fragments (F1 and F2, Fig. 3A) derived from the cleavage of D3A by vEP with sizes similar to those of the p17 had the same N-terminal sequence of $\mathrm{Ile}^{20}-\mathrm{Ile}-\mathrm{His}-\mathrm{Gly}-\mathrm{Ser}^{24}$. Therefore Lys ${ }^{19}-$ Ile $^{20}$ was one of the sites within D3A cleaved by vEP, and since both F1 and F2 had the same N-terminal 
A
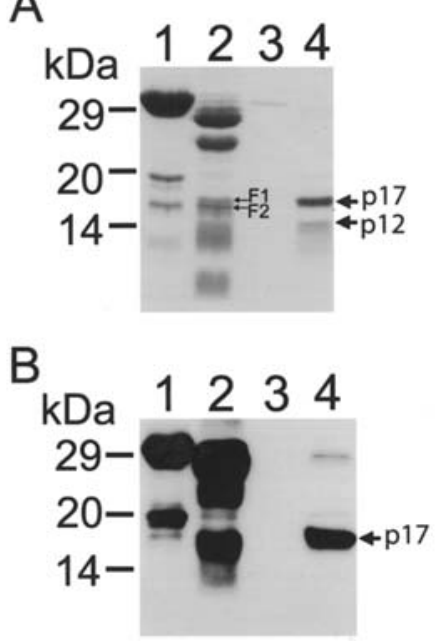

Figure 3. SDS-PAGE and Western blot analysis of vEP-cleaved D3A. Purified D3A $(10 \mu \mathrm{g})$ was incubated with vEP (40 $\mathrm{ng})$ in a $20 \mu 1$ reaction volume for $10 \mathrm{~min}$ at room temperature. The reaction was terminated with 1,10-phenanthroline $(1 \mathrm{mM})$ followed by addition of $20 \mu 1$ of $2 \mathrm{X}$ SDSPAGE sample buffer. The sample $(30 \mu 1)$ was subjected to SDS-PAGE (A) while the rest was subjected to Western blot analysis with antibody against the large subunit of caspase-3 (B). Lanes 1, non-activated D3A; 2, D3A activated with vEP; 3 , vEP only; 4, mature wild-type caspase-3. F1 and F2 indicate fragments where $\mathrm{N}$-terminal sequences have been determined.

sequence but had different molecular masses, they could have different $\mathrm{C}$-terminal sequences, suggesting a cleavage occurring probably near the residue corresponding to Asp ${ }^{175}$. Unfortunately, sequencing data were not successfully obtained to reveal the cleavage at the p17 and p12 junction of D3A.

Cleavage of PARP. PARP is an important 116-kDa zinc-finger protein for DNA repair, transcription and chromosomal stability (20). Caspase-3 is known to cleave PARP during apoptosis to generate 85 - and $24-\mathrm{kDa}$ fragments. Addition of D3A to a cell-free extract prepared from NIH3T3 cells resulted in the cleavage of PARP (Fig. 4). Since D3A has some activity toward the peptide substrate, the cleavage of PARP in cell-free extract by D3A was not unexpected. However, if D3A was first treated with vEP and then added to the cell-free extract, more cleavage of PARP was observed for the same incubation time (Fig. 4). This increase in the amount of PARP cleavage confirms that D3A activated by vEP also retained the same substrate specificity as mature caspase-3. Cleavage of PARP has become a standard way to identify the presence of caspase- 3 and its activity. The mature caspase- 3 resulted from the cleavage of D3A by vEP, yet still preserved the same specificity despite being processed at a different cleavage site.

\section{Discussion}

Activation of procapase-3 by other proteases has previously been reported for enzymes such as cytotoxic $\mathrm{T}$ cell serine protease granzyme B $(21,22)$ or other interleukin-1Bconverting enzyme (ICE)-like proteases (23). Zhou and Salvesen (24) studied the activation of procaspase- 7 by various serine proteases covering a cross-section of the specificity

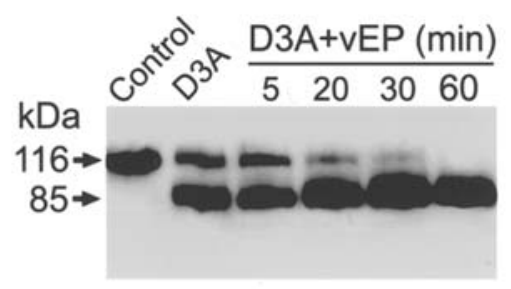

Figure 4. Cleavage of PARP by vEP-activated D3A. D3A $(0.5 \mathrm{mg} / \mathrm{ml})$ was incubated without or with vEP $(2 \mu \mathrm{g} / \mathrm{ml})$ at room temperature for $10 \mathrm{~min}$, and the reaction was terminated with 1,10-phenanthrolin $(1 \mathrm{mM})$. Ten $\mu 1$ of cell lysate $(150 \mu \mathrm{g}$ protein) was incubated with $10 \mu 1$ of non-activated D3A for $30 \mathrm{~min}$ or with $10 \mu 1$ of vEP-activated D3A for 5, 20, 30 and $60 \mathrm{~min}$ at room temperature. The control sample contained only cell lysate incubated for $30 \mathrm{~min}$ at room temperature.

repertoire of proteolysis and showed that the cleavage of the zymogen occurred within the linker region between the large and small subunits converting a single-chain zymogen to two-chain forms. vEP is a broad specificity protease which can cleave at the carboxyl side of Asp, Thr and Tyr, with the surrounding amino acid sequence playing an important part in the cleavage sites (5). A particular feature among the various known cleavage sites of vEP is the presence of a hydrophobic amino acid which is immediately adjacent to the point of cleavage. Although we have no data to show the structural change (if any) that might possibly be incurred by the Ala substitutions in D3A, we do not think that the ability of vEP to cleave D3A and activate it was afforded by the structural change produced by these substitutions. Instead, the formation of active caspase- 3 from D3A was due more to the cleavage at sites that are near $\mathrm{Asp}^{28}$ and $\mathrm{Asp}^{175}$.

It is known that expression of caspase zymogens in $E$. coli leads to their activation, a process that is thought to be the consequence of intrinsic proteolytic activity residing in the caspase zymogens. This autoprocessing of the zymogens to active forms has been dubbed the induced-proximity model, which states that caspase zymogens are autoprocessed once they are brought into proximity with each other (25). As D3A is an uncleavable form of procaspase-3, the problem associated with autoprocessing was largely avoided. D3A has previously been shown to be catalytically competent but the catalytic efficiency is $\sim 130$ - to 200 -fold lower than its mature form despite having similar $K_{\mathrm{m}}$ values $(14,15)$. Although the crystal structure of caspase- 3 has been determined $(26,27)$, no crystal structure for procaspase- 3 is yet available. However, the crystal structure obtained for procaspase-7 (28) shows that the presence of cleavage at the junction between the large and small subunits is necessary to form the correct active site for binding to substrate. It appears that the retainment of a correct active conformation by the processed enzyme is crucial for maintaining substrate specificity even though the zymogen might not be cleaved at the precise cleavage site. This is a significant event that emphasizes the versatility of a protease which has broad specificity, whereby random cleavage of a zymogen leads to an active enzyme that further amplifies a particular reaction cascade in vivo.

Here we have shown the activation of a procaspase- 3 mutant that is incapable of self-activating due to the absence of the critical aspartate residues in the cleavage sites. The 
activation of procaspase- 3 exhibited a similar feature with that of prothrombin activation (5) in that the mature enzyme was further subjected to rapid degradation by vEP. These activation events suggest that zymogen activation by vEP in both procaspase- 3 and prothrombin may not be a specific reaction, but could be a by-product arising from the result of cleavage of the zymogen at sites close to those recognized by their natural activators. This property of vEP is largely attributed to its broad specificity. Although the activation may not be a significant event in the case of wild-type procaspase- 3 , which is capable of auto-activation, this finding shows that the cleavage site recognized by vEP does not converge on the specificity associated with the critical aspartate residues essential for auto-activation.

There is currently no evidence to suggest that activation of procaspase- 3 by vEP occurs inside the cell. In order for vEP to have any activity toward procaspase- 3 it needs to enter the cell. There is yet no direct evidence to show that entrance of vEP into the cell actually takes place. However, confocal microscopy images of NIH3T3 cells treated with $5 \mu \mathrm{g} / \mathrm{ml}$ fluorescein-labeled recombinant vEP indicates some penetration of vEP into the cells after a treatment time of $1 \mathrm{~h}$ (data not shown). The activation of procaspase- 3 by vEP as described here emphasizes the ability of vEP (as a protease) to activate different types of zymogens rather than being a direct link to apoptosis, and certainly by no means suggests that vEP activates procaspase-3 and causes cell death. Furthermore, the activation of procaspase- 3 is a response to the cell death pathway and not a cause of cell death. However, the ability of vEP to activate certain proteases through cleavage of the respective zymogens does make it a formidable enzyme to be present in the host body during $V$. vulnificus infection. The in vitro activation described here extends the capability of the $V$. vulnificus extracellular metalloprotease with respect to zymogen activation, and also opens up new ground for future investigation into the function of this broad specificity protease.

\section{Acknowledgements}

This study was supported by the ERC program of MOST/ KOSEF through Research Center for Proteineous Materials (RCPM) of Chosun University, and by research funds from Chosun University, 2003.

\section{References}

1. Chiang SR and Chuang YC: Vibrio vulnificus infection: clinical manifestations, pathogenesis, and antimicrobial therapy. J Microbiol Immunol Infect 36: 81-88, 2003.

2. Ulusarac $\mathrm{O}$ and Carter E: Varied clinical presentations of Vibrio vulnificus infections: a report of four unusual cases and review of the literature. South Med J 97: 163-168, 2004.

3. Miyoshi S, Oh EG, Hirata K and Shinoda S: Exocellular toxic factors produced by Vibrio vulnificus. J Toxicol Toxin Rev 12: 253-288, 1993.

4. Miyoshi S and Shinoda S: Microbial metalloproteases and pathogensis. Microbes Infect 2: 91-98, 2000

5. Chang AK, Kim HY, Park JE, Acharya P, Park I-S, Yoon SM, You HJ, Hahm K-S, Park JK and Lee JS: Vibrio vulnificus secretes a broad-specificity metalloprotease capable of interfering with blood homeostasis through prothrombin activation and fibrinolysis. J Bacteriol 187: 6909-6916, 2005.

6. Jacobson MD, Weil M and Raff MC: Programmed cell death in animal development. Cell 88: 347-354, 1997.
7. Danial NN and Korsmeyer SJ: Cell death: critical control points. Cell 116: 205-219, 2004

8. Shi Y: Mechanisms of caspase activation and inhibition during apoptosis. Mol Cell 9: 459-470, 2002.

9. Riedl SJ and Shi Y: Molecular mechanisms of caspase regulation during apoptosis. Nat Rev Mol Cell Biol 5: 897-907, 2004.

10. Maier JK, Lahoua Z, Gendron NH, Fetni R, Johnston A, Davoodi J, Rasper D, Roy S, Slack RS, Nicholson DW and MacKenzie AE: The neuronal apoptosis inhibitory protein is a direct inhibitor of caspases 3 and 7. J Neurosci 22: 2035-2043, 2002.

11. Salvesen GS and Duckett CS: IAP proteins: blocking the road to death's door. Nat Rev Mol Cell Biol 3: 401-410, 2002.

12. Kuida K, Zheng TS, Na S, Kuan C, Yang D, Karasuyama H, Rakic P and Flavell RA: Decreased apoptosis in the brain and premature lethality in CPP32-deficient mice. Nature 384: 368-372, 1996

13. Han Z, Hendrickson EA, Bremner TA and Wyche JH: A sequential two-step mechanism for the production of the mature p17:p12 form of caspase-3 in vitro. J Biol Chem 272: 13432-13436, 1997.

14. Bose K, Pop C, Feeney B and Clark AC: An uncleavable procaspase- 3 mutant has a lower catalytic efficiency but an active site similar to that of mature caspase-3. Biochemistry 42 : 12298-12310, 2003

15. Karki P, Lee JS, Shin SY, Cho B and Park I-S: Kinetic comparison of procaspase-3 and caspase-3. Arch Biochem Biophys 442: 125-132, 2005.

16 Laemmli UK: Cleavage of structural proteins during the assembly of the head of bacteriophage T4. Nature 227: 680-685, 1970.

17. Orth $\mathrm{K}$ and $\mathrm{O}$ 'Rourke $\mathrm{K}$ : Molecular ordering of apoptotic mammalian CDE-3/ICE-like proteases. J Biol Chem 271: 20977-20980, 1996.

18. Stennicke HR and Salvesen GS: Biochemical characteristics of caspase-3, -6, -7 and -8. J Biol Chem 272: 25719-25723, 1997.

19. Sun J, Bottomley SP, Kumar S and Bird PI: Recombinant caspase-3 expressed in Pichia pastoris is fully activated and kinetically indistinguishable from the native enzyme. Biochem Biophys Res Commun 238: 920-924, 1997.

20. D'Amours D, Desnoyers S, D'Silva I and Poirier GG: Poly (ADP-ribosyl)ation reactions in the regulation of nuclear functions. Biochem J 342: 249-268, 1999.

21. Darmon AJ, Nicholson DW and Bleackley RC: Activation of the apoptotic protease CPP32 by cytotoxic T-cell-derived granzyme B. Nature 377: 446-448, 1995.

22. Quan LT, Tewari M, O'Rourke K, Dixit V, Snipas SJ, Poirier GG, Ray C, Pickup DJ and Salvesen GS: Proteolytic activation of the cell death protease Yama/CPP32 by granzyme B. Proc Natl Acad Sci USA 93: 1972-1976, 1996.

23. Liu X, Kim CN, Pohl J and Wang X: Purification and characterization of an interleukin-1ß-converting enzyme family protease that activates cysteine protease P32 (CPP32). J Biol Chem 271: 13371-13376, 1996.

24. Zhou Q and Salvesen GS: Activation of pro-caspase-7 by serine proteases includes a non-canonical specificity. Biochem J 324: 361-364, 1997.

25. Salvesen GS and Dixit VM: Caspase activation: the inducedproximity model. Proc Natl Acad Sci USA 96: 10964-10967, 1999.

26. Mittl PR, Di-Marco S, Krebs JF, Bai X, Karanewsky DS, Priestle JP, Tomaselli KJ and Grutter MG: Structure of recombinant human CPP32 in complex with the tetrapeptide acetyl-Asp-Val-Ala-Asp fluoromethyl ketone. J Biol Chem 272: 6539-6547, 1997.

27. Rotonda J, Nicholson DW, Fazil KM, Gallant M, Gareau Y, Labelle M, Peterson EJ, Rasper DM, Ruel R, Vaillancourt JP, Thornberry NA and Becker JW: The three-dimensional structure of apopain/CPP32, a key mediator of apoptosis. Nat Struct Biol 3: 619-625, 1996.

28. Chai J, Wu Q, Shiozaki E, Srinivasula SM, Alnemri ES and Shi Y: Crystal structure of a procaspase-7 zymogen: mechanisms of activation and substrate binding. Cell 107: 399-407, 2001. 